\title{
Efficient synthesis of chiral homodimeric 4-(N,N-dimethylamino)pyridine carbamate derivatives
}

\author{
Eduardo Busto, Vicente Gotor-Fernández, and Vicente Gotor* \\ Departamento de Química Orgánica e Inorgánica and Instituto Universitario de Biotecnología \\ de Asturias. Universidad de Oviedo. 33071- Oviedo (Asturias). Spain \\ E-mail: vgs@fq.uniovi.es
}

Dedicated to Benito Alcaide in occasion of his 60th birthday

DOI: http://dx.doi.org/10.3998/ark.5550190.0011.311

\begin{abstract}
The synthesis of a novel family of optically active 4-( $N, N$-dimethylamino)pyridine carbamate derivatives has been achieved with excellent isolated yields using different linear diamines as starting materials to produce dimeric compounds of variable length. Optically active homodimeric 4-( $N, N$-dimethylamino)pyridine carbamate derivatives obtained through this chemoenzymatic route present interesting structural properties as possible ligands or nucleophilic catalysts in asymmetric catalysis.
\end{abstract}

Keywords: 4-(N,N-Dimethylamino)pyridine, carbamates, chirality, ligands

\section{Introduction}

Pyridine derivatives are a family of organic compounds from the aromatic heterocyclic series characterized by a six-member ring structure composed of five carbons atoms and one nitrogen atom. Probably two of the most attractive pyridine structures are 4-( N,N-dimethylamino)pyridine (DMAP, 1) and 4-pyrrolidinopyridine (PPY, 2), which are probably the most useful of all nucleophilic catalysts providing high rate acceleration in numerous organic reactions, ${ }^{1}$ and their catalytic efficiency has encouraged, in recent years, the synthesis of chiral derivatives in order to develop stereoselective transformations. ${ }^{2}$ 
<smiles>CN(C)c1ccncc1</smiles>

$\operatorname{DMAP}(\mathbf{1})$<smiles>c1cc(N2CCCC2)ccn1</smiles>

PPY (2)

Figure 1. Structure of DMAP and PPY

Planar-chiral catalysts derived from DMAP catalyze a wide variety of asymmetric processes based in the $\pi$-complexation of a heterocycle to a transition metal. ${ }^{3}$ Introduction of chirality in a stereogenic center of the substituents of the pyridine ring has been studied over the last decades, observing that those which are far from the active heterocyclic nitrogen posses higher reactivity than those which present the chirality closed to the nitrogen reactive atom, as occurs when a stereogenic center is located in the 3-position of the pyridine ring instead of C-2. ${ }^{4}$ However 2substituted DMAP derivatives are generally more powerful stereoselective catalysts although they have been scarcely studied due to the difficult chemical access to this position. ${ }^{5}$

At the same time different pyridine based macrocycles have been synthesized by different research groups presenting interesting properties as chiral ligands ${ }^{6}$ or chiral solvating agents. ${ }^{7}$ Here we report the synthesis of novel dimeric carbamate derivatives of variable length $[(S, S)-4 a-$ c] depending on the lineal diamine employed in the synthetic route. To this aim (1S)-(-)-1-(4chloropyridin-2-yl)ethanol $(S)-\mathbf{3}$ has been selected as an ideal precursor of this class of compounds (Scheme 1).

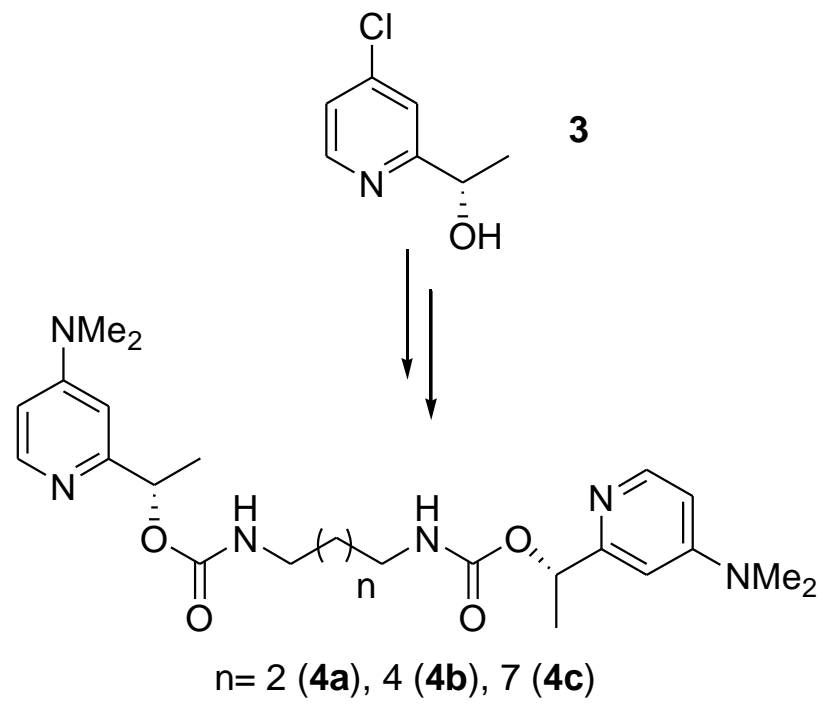

Scheme 1. Use of the alcohol derivative $(S)$-3 as a precursor of dimers $(S, S)-\mathbf{4 a - c}$. 


\section{Results and Discussion}

Biotransformations are well-recognized processes that allow the synthesis of enantiomerically pure compounds under very mild reaction conditions. ${ }^{8}$ Use of lipases allows the preparation of optically active compounds such as esters, alcohols, amines or amides starting from racemic or meso-compounds, which can be easily converted into other functional groups as carbamates by classical chemical synthesis. Carbamates are of value in medicinal chemistry because this functionality is part of various therapeutic agents allowing a better permeability across the cellular membranes. ${ }^{9}$ In fact our research group have previously reported the preparation of nucleoside carbamates ${ }^{10}$ and vitamin $\mathrm{D}_{3}$ derivatives ${ }^{11}$ by reaction of the corresponding carbonates with different amines.

In our ongoing project for the development of chiral DMAP derivatives, we have previously described the development of a chemoenzymatic synthesis for the production of $(S)-4-(N, N-$ dimethylamino)-2-(1-hydroxyalkyl)pyridines. Pseudomonas cepacia lipase or different alcohol dehydrogenases (ADHs) are responsible for the stereoselective processes in the synthesis of the corresponding (S)-4-chloro-2-(1-hydroxyalkyl)pyridines, using chiral DMAP analogues with interesting applications as enantioselective alkoxycarbonylating agents or chiral ligands in the addition of diethylzinc to benzaldehyde (Scheme 2). ${ }^{12}$<smiles>CC(O)c1cc(Cl)ccn1</smiles>

rac-3

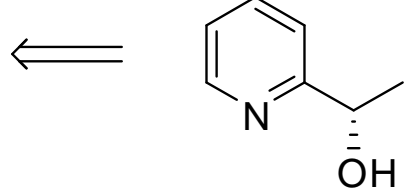

(S) -3<smiles>CC(=O)c1cc(Cl)ccn1</smiles>

5

Scheme 2. Racemic alcohol and prochiral ketone, precursors of alcohol $(S)$-3 by enzymatic methods using lipases or ADHs.

A remarkable advantage of this type of precursors is the versatility of the chlorine atom located in the 4-position of the pyridine ring, allowing the functionalization of the heterocycle by simple nucleophilic substitution reactions. This possibility constitutes a window of opportunities for the preparation of interesting macrocycles by just designing adequate cyclization strategies. For instance in this field, the preparation of different symmetrical and unsymmetrical bispyridines has been described, which have already been used for the synthesis of shapepersistent macrocycles. ${ }^{13}$

At this point, and because of the versatility of the vinyloxycarbonyl group, we decided to react $(S)$-3 with vinyl chloroformate 6 obtaining the enantiopure carbonate $(S)-7$ with $87 \%$ yield (Scheme 3). No racemization was observed in the process by comparing the HPLC 
chromatogram of $\mathbf{7}$ and the corresponding racemic carbonate synthesized in the same manner from the racemic alcohol.

(S)-3

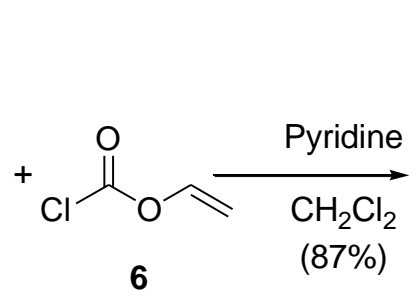<smiles>C/C=C\OC(=O)OC(C)c1cc(Cl)ccn1</smiles>

(S)-7 O

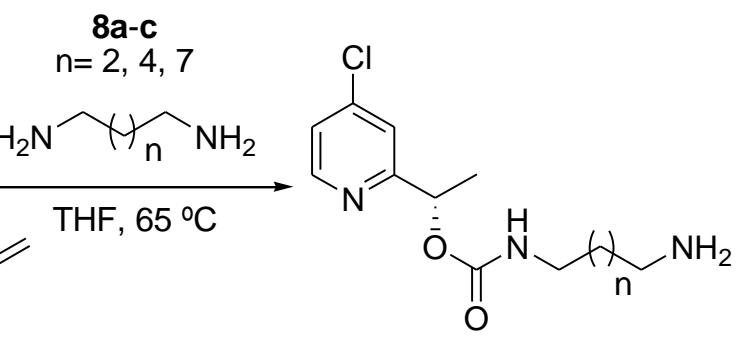

(S)-9a (89\%), 9b (86\%), 9c (80\%)

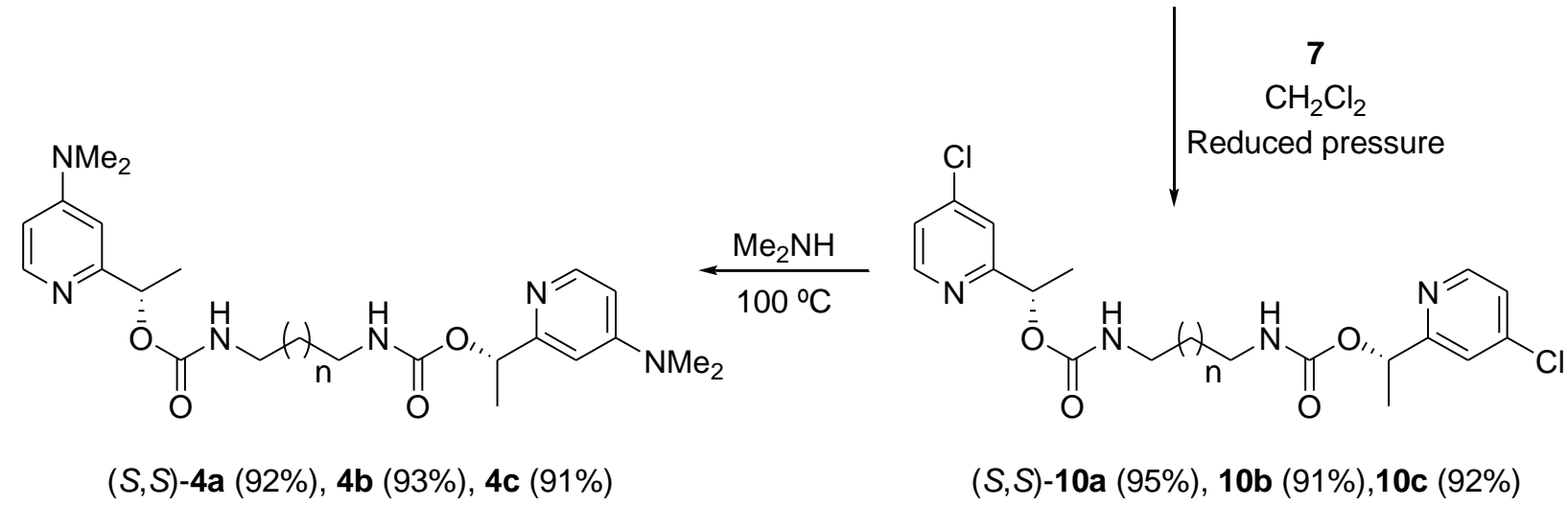

Scheme 3. Chemical synthesis of $(S, S)-\mathbf{4 a}-\mathbf{c}$.

Reaction of pyridine 7 with different linear diamines of different length between the two amino groups (1,4-butanediamine 8a, 1,6-hexanediamine $\mathbf{8 b}$ and 1,9-nonanediamine 8c) in THF at $65{ }^{\circ} \mathrm{C}$ led to the isolation of the corresponding carbamates $(S)-9 \mathbf{a}-\mathbf{c}$ in high yield (80-89\%) after $14 \mathrm{~h}$. At this point the carbamate $(S)$-9a was reacted with vinyl carbonate $(S)$-7 in 1,4dioxane however no reaction was observed at $80{ }^{\circ} \mathrm{C}$ after $22 \mathrm{~h}$, for that reason temperature was increased to $100^{\circ} \mathrm{C}$ and DMAP was added as catalyst, but after additional $2 \mathrm{~h}$ no reaction was observed. Surprisingly when the reaction was stopped and the solvent evaporated at reduced pressure, the ${ }^{1} \mathrm{H}$ NMR spectrum of the crude reaction showed a $70 \%$ conversion to $(S, S)-\mathbf{1 0 a}$, which means that the reaction evolved to the final product in the rotary evaporator.

In view of this result we decided to react in a different experiment, the intimate mixture of carbonate 7 and carbamate $(S)-(9 a)$ for $1 \mathrm{~h}$ at room temperature, TLC analysis showed the appearance of $(S, S)-\mathbf{1 0 a}$ in approximately $70 \%$ yield. To optimize the process for the production of the desired carbamate, we finally decided to prepare a solution of $(S)-7$ and $(S)-9 \mathbf{a}$ in $\mathrm{CH}_{2} \mathrm{Cl}_{2}$ and distilled the solvent until dryness, repeating the cycle 3 times, and obtaining the dimer $(S, S)$ 10a in an excellent isolated yield (95\%). The same approach was performed using carbamates $(S)-9 b$ and $(S)$-9c affording after flash chromatography the corresponding dimers in $91 \%$ and $92 \%$ yield respectively. 
The presence of the two chlorine atoms offers interesting possibilities in the field of supramolecular chemistry, as they can be used as linkage points for suitable functionalities. In our case we decided to carry out the synthesis of DMAP derivatives heating at $100{ }^{\circ} \mathrm{C}$ the corresponding synthons with a $40 \%$ aqueous solution of dimethylamine obtaining the dimers $(S, S)-4 a-c$ in excellent yields (91-93\%) after $24 \mathrm{~h}$. To ensure that no racemization occurred during the process, and in the same manner that it was done for $\mathbf{7}$, the chiral compound and the racemic product $\mathbf{4 a}$ were injected into the HPLC observing no racemization during the overall synthesis. Currently investigations of these DMAP derivatives as nucleophilic catalyst are in progress and the role of the chloro derivatives will be studied as chiral ligands and as intermediates for macrocyclic structures synthesis.

\section{Conclusions}

In summary, we have developed an easy and straightforward general synthesis of dichloro functionalized dimers $(S, S)$-10a-c, which seem to be useful building blocks for the synthesis of macrocycles. Alternatively DMAP derivatives $(S, S)-\mathbf{4 a - c}$ have been obtained by nucleophilic substitution, and these compounds could be valuable structures in asymmetric organocatalysis.

\section{Experimental Section}

General Procedures. All reagents were purchased from commercial suppliers and were used without further purification. Solvents were distilled over an adequate desiccant and stored under nitrogen. Flash chromatography was performed using Merck silica gel 60 (230-400 mesh). Melting points were taken using a Gallenkamp apparatus and are uncorrected. Specific optical rotations were measured using a Perkin-Elmer 241 polarimeter and are quoted in units of deg. $\mathrm{cm}^{2} \cdot \mathrm{g}^{-1}$. IR spectra were recorded on using $\mathrm{NaCl}$ plates or $\mathrm{KBr}$ pellets in a Perkin-Elmer 1720-X F7. NMR spectra were obtained using AC-200 $\left({ }^{1} \mathrm{H}, 200.13 \mathrm{MHz}\right.$ and $\left.{ }^{13} \mathrm{C}, 50.3 \mathrm{MHz}\right)$, AC-300 $\left({ }^{1} \mathrm{H}, 300.13 \mathrm{MHz}\right.$ and $\left.{ }^{13} \mathrm{C}, 75.5 \mathrm{MHz}\right), \mathrm{DPX}-300\left({ }^{1} \mathrm{H}, 300.13 \mathrm{MHz}\right.$ and $\left.{ }^{13} \mathrm{C}, 75.5 \mathrm{MHz}\right)$ or AV-400 $\left({ }^{1} \mathrm{H}, 400.13 \mathrm{MHz}\right.$ and $\left.{ }^{13} \mathrm{C}, 100.6 \mathrm{MHz}\right)$ spectrometers. Mass spectra were recorded on a Hewlett-Packard 1100 LC/MSD. Microanalyses were performed on a Perkin-Elmer model 2400 instrument (Universidad de Oviedo, Departamento de Química Orgánica e Inorgánica, Spain).

(1S)-(-)-1-(4-Chloropyridin-2-yl)ethanol (3). The preparation of 3 was done according to the procedure previously reported in the literature. ${ }^{12}$

(1S)-(-)-1-(4-Chloropyridin-2-yl)ethyl vinyl carbonate (7). To a solution of ( $S$ )-3 (52 mg, 0.33 $\mathrm{mmol})$ in dry $\mathrm{CH}_{2} \mathrm{Cl}_{2}(2.6 \mathrm{~mL})$ were successively added pyridine $(29 \mu \mathrm{L}, 0.36 \mathrm{mmol})$ and vinyl chloroformate $(33 \mu \mathrm{L}, 0.36 \mathrm{mmol})$ in small portions at $0{ }^{\circ} \mathrm{C}$. The reaction was stirred at room 
temperature during $18 \mathrm{~h}$ until complete consumption of the starting material. The solvent was evaporated and the residue purified by flash chromatography (10\% EtOAc/hexane) yielding 70 mg of $(S)-7$ as a colorless oil (93\%). $R_{f}\left(10 \%\right.$ EtOAc/hexane): $0.29 ;[\alpha]_{\mathrm{D}}{ }^{20}=-28.0(\mathrm{c}=1.5$, $\left.\mathrm{CHCl}_{3}\right)$; IR $(\mathrm{NaCl}) v_{\max } 1650,1258 \mathrm{~cm}^{-1} ;{ }^{1} \mathrm{H}-\mathrm{NMR}\left(\mathrm{CDCl}_{3}, 300.13 \mathrm{MHz}\right): \delta 1.69\left(\mathrm{~d},{ }^{3} J_{\mathrm{HH}}=6.7\right.$, $3 \mathrm{H}), 4.63\left(\mathrm{dd},{ }^{3} J_{\mathrm{HH}}=6.2 \mathrm{~Hz},{ }^{2} J_{\mathrm{HH}}=2.0 \mathrm{~Hz}, 1 \mathrm{H},\right), 4.98\left(\mathrm{dd},{ }^{3} J_{\mathrm{HH}}=13.8 \mathrm{~Hz},{ }^{2} J_{\mathrm{HH}}=2.2 \mathrm{~Hz}, 1 \mathrm{H}\right)$, $5.84\left(\mathrm{q},{ }^{3} J_{\mathrm{HH}}=6.7 \mathrm{~Hz}, 1 \mathrm{H}\right), 7.10\left(\mathrm{dd},{ }^{3} J_{\mathrm{HH}}=13.8 \mathrm{~Hz},{ }^{3} J_{\mathrm{HH}}=6.2 \mathrm{~Hz}, 1 \mathrm{H}\right), 7.27\left(\mathrm{dd},{ }^{3} J_{\mathrm{HH}}=5.4\right.$ $\left.\mathrm{Hz},{ }^{4} J_{\mathrm{HH}}=1.9 \mathrm{~Hz}, 1 \mathrm{H}\right), 7.44\left(\mathrm{~d},{ }^{4} J_{\mathrm{HH}}=1.9 \mathrm{~Hz}, 1 \mathrm{H}\right), 8.51\left(\mathrm{~d},{ }^{3} J_{\mathrm{HH}}=5.4 \mathrm{~Hz}, 1 \mathrm{H}\right) ;{ }^{13} \mathrm{C}-\mathrm{NMR}$ $\left(\mathrm{CDCl}_{3}, 75.5 \mathrm{MHz}\right): \delta 20.8\left(\mathrm{CH}_{3}\right), 77.6(\mathrm{CH}), 98.2\left(\mathrm{CH}_{2}\right), 120.6(\mathrm{CH}), 123.4(\mathrm{CH}), 142.6(\mathrm{CH})$, $145.1(\mathrm{C}), 150.3(\mathrm{CH}), 152.0(\mathrm{C}), 161.2(\mathrm{C}) ; \mathrm{MS}\left(\mathrm{ESI}^{+}, \mathrm{m} / \mathrm{z}\right): 230\left[\left(\mathrm{M}^{37} \mathrm{Cl}+\mathrm{H}\right)^{+}, 32 \%\right], 228$ $\left[\left(\mathrm{M}^{35} \mathrm{Cl}+\mathrm{H}\right)^{+}, 100 \%\right]$; Anal. Calcd. (\%) for $\mathrm{C}_{10} \mathrm{H}_{10} \mathrm{NO}_{3} \mathrm{Cl}: \mathrm{C}, 52.76$; $\mathrm{H}, 4.43 \mathrm{~N}, 6.15$. Found: $\mathrm{C}$, $52.8 \mathrm{H}, 4.5 \mathrm{~N}, 6.2$.

\section{General procedure for the synthesis of carbamates $(S)-9$ a-c}

To a solution of $(S)-7(100 \mathrm{mg}, 0.44 \mathrm{mmol})$ in dry THF $(4 \mathrm{~mL})$ was added the corresponding amine 8a-c $(1.32 \mathrm{mmol})$. The solution was stirred at $65^{\circ} \mathrm{C}$ during $14 \mathrm{~h}$ until complete consumption of the starting material. The solvent was evaporated and the residue purified by flash chromatography affording the corresponding carbamates $(S)-9 \mathbf{a}-\mathbf{c}$ as colorless oils (80$89 \%)$.

(1S)-(-)-1-(4-Chloropyridin-2-yl)ethyl (4-aminobutyl)carbamate $(\boldsymbol{S})$-(9a). 89\% isolated yield. $R_{f}\left(50 \% \mathrm{CH}_{2} \mathrm{Cl}_{2} / 45 \% \mathrm{MeOH} / 5 \% \mathrm{NH}_{3}\right): 0.37 ;[\alpha]_{\mathrm{D}}{ }^{20}=-19.0\left(\mathrm{c}=2.0, \mathrm{CHCl}_{3}\right) ; \mathrm{IR}(\mathrm{NaCl}): v_{\max }$ 3346, 2936, 1702, 1579, 1560, 1526, 1318, 1138, $1071 \mathrm{~cm}^{-1} ;{ }^{1} \mathrm{H}-\mathrm{NMR}\left(\mathrm{CDCl}_{3}, 300.13 \mathrm{MHz}\right): \delta$ 1.39-1.80 (m, 7H), $2.28\left(\right.$ br s, 2H), $2.68\left(\mathrm{t},{ }^{3} J_{\mathrm{HH}}=6.3 \mathrm{~Hz}, 2 \mathrm{H}\right), 3.07-3.37(\mathrm{~m}, 2 \mathrm{H}), 5.39$ (br s, $1 \mathrm{H}), 5.75\left(\mathrm{q},{ }^{3} J_{\mathrm{HH}}=6.5 \mathrm{~Hz}, 1 \mathrm{H}\right), 7.16\left(\mathrm{dd},{ }^{3} J_{\mathrm{HH}}=5.4 \mathrm{~Hz},{ }^{4} J_{\mathrm{HH}}=2.0 \mathrm{~Hz}, 1 \mathrm{H}\right), 7.30\left(\mathrm{~d},{ }^{4} J_{\mathrm{HH}}=2.0\right.$ $\mathrm{Hz}, 1 \mathrm{H}), 8.42\left(\mathrm{~d},{ }^{3} \mathrm{~J}_{\mathrm{HH}}=5.4 \mathrm{~Hz}, 1 \mathrm{H}\right) ;{ }^{13} \mathrm{C}-\mathrm{NMR}\left(\mathrm{CDCl}_{3}, 75.5 \mathrm{MHz}\right): \delta 20.8\left(\mathrm{CH}_{3}\right), 27.1\left(\mathrm{CH}_{2}\right)$, $30.1\left(\mathrm{CH}_{2}\right), 40.7\left(\mathrm{CH}_{2}\right), 41.3\left(\mathrm{CH}_{2}\right), 72.5(\mathrm{CH}), 120.5(\mathrm{CH}), 122.7(\mathrm{CH}), 144.6(\mathrm{C}), 150.0(\mathrm{CH})$, $155.5(\mathrm{C}), 162.8(\mathrm{C})$; MS (ESI $\left.{ }^{+}, \mathrm{m} / \mathrm{z}\right): 274\left[\left(\mathrm{M}^{37} \mathrm{Cl}+\mathrm{H}\right)^{+}, 34 \%\right], 272\left[\left(\mathrm{M}^{35} \mathrm{Cl}+\mathrm{H}\right)^{+}, 100 \%\right]$; Anal. Calcd. (\%) for $\mathrm{C}_{12} \mathrm{H}_{18} \mathrm{~N}_{3} \mathrm{O}_{2} \mathrm{Cl}$ : C, 53.04; H, 6.68; N, 15.46. Found: C, 53.1; H, 6.7; N, 15.5.

(1S)-(-)-4-(Chloropyridin-2-yl)ethyl (6-aminohexyl)carbamate $(\boldsymbol{S})$-(9b). 86\% isolated yield. $R_{f}\left(2 \% \mathrm{NH}_{3} / 48 \% \mathrm{MeOH} / 50 \% \mathrm{CH}_{2} \mathrm{Cl}_{2}\right): 0.30 ;[\alpha]_{\mathrm{D}}{ }^{20}=-24.2\left(\mathrm{c}=2.2, \mathrm{CHCl}_{3}\right) ; \mathrm{IR}(\mathrm{NaCl}): v_{\max }$ 3356, 2933, 1702, 1579, 1558, 1468, $1255 \mathrm{~cm}^{-1}$; ${ }^{1} \mathrm{H}-\mathrm{NMR}\left(\mathrm{CDCl}_{3}, 400.13 \mathrm{MHz}\right): \delta 1.20-1.36$ $(\mathrm{m}, 4 \mathrm{H}), 1.38-1.47(\mathrm{~m}, 4 \mathrm{H}), 1.51\left(\mathrm{~d},{ }^{3} J_{\mathrm{HH}}=6.5 \mathrm{~Hz}, 1 \mathrm{H}\right), 2.52($ br s, $2 \mathrm{H}), 2.65\left(\mathrm{t},{ }^{3} J_{\mathrm{HH}}=6.5 \mathrm{~Hz}\right.$, $2 \mathrm{H}), 3.05-3.21(\mathrm{~m}, 2 \mathrm{H}), 5.15(\mathrm{br} \mathrm{s}, 1 \mathrm{H}), 5.75\left(\mathrm{q},{ }^{3} J_{\mathrm{HH}}=6.6 \mathrm{~Hz}, 1 \mathrm{H}\right), 7.16\left(\mathrm{dd},{ }^{3} J_{\mathrm{HH}}=5.4 \mathrm{~Hz}\right.$, $\left.{ }^{4} J_{\mathrm{HH}}=1.7 \mathrm{~Hz}, 1 \mathrm{H}\right), 7.30\left(\mathrm{~d},{ }^{4} J_{\mathrm{HH}}=1.7 \mathrm{~Hz}, 1 \mathrm{H}\right), 8.42\left(\mathrm{~d},{ }^{3} J_{\mathrm{HH}}=5.4 \mathrm{~Hz}, 1 \mathrm{H}\right) ;{ }^{13} \mathrm{C}-\mathrm{NMR}\left(\mathrm{CDCl}_{3}\right.$, 100.6 MHz): $\delta 20.7\left(\mathrm{CH}_{3}\right), 26.1\left(2 \mathrm{CH}_{2}\right), 29.5\left(\mathrm{CH}_{2}\right), 32.8\left(\mathrm{CH}_{2}\right), 40.6\left(\mathrm{CH}_{2}\right), 41.5\left(\mathrm{CH}_{2}\right), 72.3$ $(\mathrm{CH}), 120.4(\mathrm{CH}), 122.5(\mathrm{CH}), 144.4(\mathrm{C}), 149.8(\mathrm{CH}), 155.3(\mathrm{C}), 162.6(\mathrm{C}) ; \mathrm{MS}\left(\mathrm{ESI}^{+}, \mathrm{m} / \mathrm{z}\right)$ : $302\left[\left(\mathrm{M}^{37} \mathrm{Cl}+\mathrm{H}\right)^{+}, 34 \%\right], 300\left[\left(\mathrm{M}^{35} \mathrm{Cl}+\mathrm{H}\right)^{+}, 100 \%\right]$; Anal. Calcd. (\%) for $\mathrm{C}_{14} \mathrm{H}_{22} \mathrm{~N}_{3} \mathrm{O}_{2} \mathrm{Cl}$ : C, 56.09; H, 7.40; N, 14.02. Found: C, 56.1; H, 7.5; N, 14.0.

(1S)-(-)-4-(Chloropyridin-2-yl)ethyl (9-aminononyl)carbamate $(\boldsymbol{S})$-(9c). 80\% isolated yield.

$R_{f}\left(1 \% \mathrm{NH}_{3} / 49 \% \mathrm{MeOH} / 50 \% \mathrm{CH}_{2} \mathrm{Cl}_{2}\right): 0.30 ;[\alpha]_{\mathrm{D}}{ }^{20}=-25.5\left(\mathrm{c}=1.5, \mathrm{CHCl}_{3}\right) ; \mathrm{IR}(\mathrm{NaCl}): v_{\max }$ 3336, 2928, 2855, 1702, 1578, 1558, 1467, 1255, $1138 \mathrm{~cm}^{-1} ;{ }^{1} \mathrm{H}-\mathrm{NMR}\left(\mathrm{CDCl}_{3}, 400.13 \mathrm{MHz}\right): \delta$ 
1.18-1.29 (m, 10H), 1.33-1.46 (m, 4H), $2.65\left(\mathrm{t},{ }^{3} J_{\mathrm{HH}}=7.0 \mathrm{~Hz}, 3 \mathrm{H}\right), 2.96$ (br s, 2H), 3.05-3.17 (m, $2 \mathrm{H}), 5.20(\mathrm{br} \mathrm{s}, 1 \mathrm{H}), 5.74\left(\mathrm{q},{ }^{3} J_{\mathrm{HH}}=6.6 \mathrm{~Hz}, 1 \mathrm{H}\right), 7.15\left(\mathrm{dd},{ }^{3} J_{\mathrm{HH}}=5.3 \mathrm{~Hz},{ }^{4} J_{\mathrm{HH}}=1.9 \mathrm{~Hz}, 1 \mathrm{H}\right)$, $7.30\left(\mathrm{~d},{ }^{4} J_{\mathrm{HH}}=1.9 \mathrm{~Hz}, 1 \mathrm{H}\right), 8.41\left(\mathrm{~d},{ }^{3} J_{\mathrm{HH}}=5.3 \mathrm{~Hz}, 1 \mathrm{H}\right) ;{ }^{13} \mathrm{C}-\mathrm{NMR}\left(\mathrm{CDCl}_{3}, 100.6 \mathrm{MHz}\right): \delta 21.0$ $\left(\mathrm{CH}_{3}\right), 26.7\left(3 \mathrm{CH}_{2}\right), 29.3\left(2 \mathrm{CH}_{2}\right), 29.8\left(\mathrm{CH}_{2}\right), 32.7\left(\mathrm{CH}_{2}\right), 41.0\left(\mathrm{CH}_{2}\right), 41.7\left(\mathrm{CH}_{2}\right), 72.5(\mathrm{CH})$, $120.3(\mathrm{CH}), 122.8(\mathrm{CH}), 144.7(\mathrm{C}), 150.1(\mathrm{CH}), 155.6(\mathrm{C}), 162.9(\mathrm{C}) ; \mathrm{MS}\left(\mathrm{ESI}^{+}, \mathrm{m} / \mathrm{z}\right): 344$ $\left[\left(\mathrm{M}^{37} \mathrm{Cl}+\mathrm{H}\right)^{+}, 33 \%\right], 342\left[\left(\mathrm{M}^{35} \mathrm{Cl}+\mathrm{H}\right)^{+}, 100 \%\right]$; Anal. Calcd. (\%) for $\mathrm{C}_{17} \mathrm{H}_{28} \mathrm{~N}_{3} \mathrm{O}_{2} \mathrm{Cl}: \mathrm{C}, 59.72 ; \mathrm{H}$, 8.26; N, 12.29. Found: C, 59.7; H, 8.3; N, 12.0.

\section{General procedure for the synthesis of dimers $(S, S)-(10 \mathrm{a}-\mathrm{c})$}

The corresponding carbamate $(S)-9 \mathbf{a}-\mathbf{c}(0.38 \mathrm{mmol})$ and $(S)-7(80 \mathrm{mg}, 0.35 \mathrm{mmol})$ were mixed in a round-bottom flask and the mixture was dissolved in $\mathrm{CH}_{2} \mathrm{Cl}_{2}(1.8 \mathrm{~mL})$. The solvent was evaporated under reduced pressure at low temperature observing partial formation of the final product by TLC analysis. This process was repeated 3 times until complete consumption of the carbonate $(S)$-7. The crude was purified by flash chromatography yielding the corresponding dimers $(S, S)-\mathbf{1 0 a}-\mathbf{c}$ as colorless oils $(91-95 \%)$.

$(\mathbf{1 S , 1} \boldsymbol{S})$-Bis[1-(4-chloropyridin-2-yl)ethyl] butane-1,4-diylbiscarbamate $(\boldsymbol{S}, \boldsymbol{S})$-(10a). 95\% isolated yield. $R_{f}(100 \% \mathrm{EtOAc}): 0.32 ;[\alpha]_{\mathrm{D}}{ }^{20}=-19.3\left(\mathrm{c}=1.0, \mathrm{CHCl}_{3}\right) ; \mathrm{IR}(\mathrm{NaCl}): v_{\max } 3322$, 1700, 1581, 1559, 1542, 1263, $1074 \mathrm{~cm}^{-1}$; ${ }^{1} \mathrm{H}-\mathrm{NMR}\left(\mathrm{CDCl}_{3}, 300.13 \mathrm{MHz}\right): \delta 1.40-1.55(\mathrm{~m}, 4 \mathrm{H})$, $1.50\left(\mathrm{~d},{ }^{3} J_{\mathrm{HH}}=6.6 \mathrm{~Hz}, 6 \mathrm{H}\right), 3.09-3.22(\mathrm{~m}, 4 \mathrm{H}), 5.02(\mathrm{br} \mathrm{s}, 2 \mathrm{H}), 5.80\left(\mathrm{q},{ }^{3} J_{\mathrm{HH}}=6.6 \mathrm{~Hz}, 2 \mathrm{H}\right), 7.20$ $\left(\mathrm{dd},{ }^{3} J_{\mathrm{HH}}=5.1 \mathrm{~Hz},{ }^{4} J_{\mathrm{HH}}=2.0 \mathrm{~Hz}, 1 \mathrm{H}\right), 7.34\left(\mathrm{~d},{ }^{4} J_{\mathrm{HH}}=2.0 \mathrm{~Hz}, 1 \mathrm{H}\right), 8.47\left(\mathrm{~d},{ }^{3} J_{\mathrm{HH}}=5.1 \mathrm{~Hz}, 1 \mathrm{H}\right)$; ${ }^{13} \mathrm{C}-\mathrm{NMR}\left(\mathrm{CDCl}_{3}, 75.5 \mathrm{MHz}\right): \delta 20.9\left(2 \mathrm{CH}_{3}\right), 27.1\left(2 \mathrm{CH}_{2}\right), 40.5\left(2 \mathrm{CH}_{2}\right), 72.7(2 \mathrm{CH}), 120.6$ $(2 \mathrm{CH}), 122.8(2 \mathrm{CH}), 144.6(2 \mathrm{C}), 150.1(2 \mathrm{CH}), 155.5(2 \mathrm{C}), 162.7(2 \mathrm{C}) ; \mathrm{MS}\left(\mathrm{ESI}^{+}, \mathrm{m} / \mathrm{z}\right): 457$ $\left[\left(\mathrm{M}^{37} \mathrm{Cl}^{35} \mathrm{Cl}+\mathrm{H}\right)^{+}, 66 \%\right], 455\left[\left(\mathrm{M}^{35} \mathrm{Cl}^{35} \mathrm{Cl}+\mathrm{H}\right)^{+}, 100 \%\right]$; Anal. Calcd. (\%) for $\mathrm{C}_{20} \mathrm{H}_{24} \mathrm{~N}_{4} \mathrm{O}_{4} \mathrm{Cl}_{2}$ : C, 52.85; H, 5.33; N, 12.33. Found: C, 52.8; H, 5.3; N, 12.2.

$(\mathbf{1}, \mathbf{1}$ 'S)-Bis[1-(4-chloropyridin-2-yl)ethyl] hexane-1,6-diylbiscarbamate $(\boldsymbol{S}, \boldsymbol{S})$-(10b). $91 \%$ isolated yield. $R_{f}\left(80 \%\right.$ EtOAc/hexane): $0.33 ;[\alpha]_{\mathrm{D}}{ }^{20}=-27.9\left(\mathrm{c}=1.5, \mathrm{CHCl}_{3}\right)$; IR $(\mathrm{NaCl}): v_{\max }$ 3332, 2934, 1699, 1558, 1469, 1253, 1138, 1071, $829 \mathrm{~cm}^{-1} ;{ }^{1} \mathrm{H}-\mathrm{NMR}\left(\mathrm{CDCl}_{3}, 300.13 \mathrm{MHz}\right): \delta$ 1.16-1.29 (m, 4H), 1.33-1.44 (m, 4H), $1.47\left(\mathrm{~d},{ }^{3} J_{\mathrm{HH}}=6.5 \mathrm{~Hz}, 6 \mathrm{H}\right), 2.98-3.19(\mathrm{~m}, 4 \mathrm{H}), 5.20$ (br s, $2 \mathrm{H}), 5.71\left(\mathrm{q},{ }^{3} J_{\mathrm{HH}}=6.8 \mathrm{~Hz}, 2 \mathrm{H}\right), 7.17\left(\mathrm{dd},{ }^{3} J_{\mathrm{HH}}=5.4 \mathrm{~Hz},{ }^{4} J_{\mathrm{HH}}=1.7 \mathrm{~Hz}, 2 \mathrm{H}\right), 7.32\left(\mathrm{~d},{ }^{4} J_{\mathrm{HH}}=1.7\right.$ $\mathrm{Hz}, 2 \mathrm{H}), 8.43\left(\mathrm{~d},{ }^{3} \mathrm{~J}_{\mathrm{HH}}=5.4 \mathrm{~Hz}, 2 \mathrm{H}\right) ;{ }^{13} \mathrm{C}-\mathrm{NMR}\left(\mathrm{CDCl}_{3}, 75.5 \mathrm{MHz}\right): \delta 20.8\left(2 \mathrm{CH}_{3}\right), 25.9\left(2 \mathrm{CH}_{2}\right)$, $29.5\left(2 \mathrm{CH}_{2}\right), 40.5\left(2 \mathrm{CH}_{2}\right), 72.3(2 \mathrm{CH}), 120.3(2 \mathrm{CH}), 122.5(2 \mathrm{CH}), 144.5(2 \mathrm{C}), 149.8(2 \mathrm{CH})$, 155.4 (2C), 162.7 (2C); MS (ESI, $\mathrm{m} / \mathrm{z}): 485\left[\left(\mathrm{M}^{35} \mathrm{Cl}^{37} \mathrm{Cl}+\mathrm{H}\right)^{+}, 66 \%\right], 483\left[\left(\mathrm{M}^{35} \mathrm{Cl}^{35} \mathrm{Cl}+\mathrm{H}\right)^{+}\right.$, $100 \%$ ]; Anal. Calcd. (\%) for $\mathrm{C}_{22} \mathrm{H}_{28} \mathrm{~N}_{4} \mathrm{O}_{4} \mathrm{Cl}_{2}$ : C, 54.75; H, 5.85; N, 11.62. Found: C, 54.7; H, 5.9; N, 11.6.

$(\mathbf{1 S , 1} \boldsymbol{S})$-Bis[1-(4-chloropyridin-2-yl)ethyl] nonane-1,9-diylbiscarbamate $(\boldsymbol{S}, \boldsymbol{S})$-(10c). $92 \%$ isolated yield. $R_{f}(60 \% \mathrm{EtOAc} /$ hexane $): 0.34 ;[\alpha]_{\mathrm{D}}{ }^{20}=-23.5\left(\mathrm{c}=2.0, \mathrm{CHCl}_{3}\right)$; IR $(\mathrm{NaCl}): v_{\max }$ 3332, 2929, 1698, 1579, 1557, 1469, 1392, 1249, 1138, $829 \mathrm{~cm}^{-1} ;{ }^{1} \mathrm{H}-\mathrm{NMR}\left(\mathrm{CDCl}_{3}, 300.13\right.$ $\mathrm{MHz}): \delta 1.16-1.35(\mathrm{~m}, 10 \mathrm{H}), 1.39-1.53(\mathrm{~m}, 4 \mathrm{H}), 1.53\left(\mathrm{~d},{ }^{3} J_{\mathrm{HH}}=6.5 \mathrm{~Hz}, 6 \mathrm{H}\right), 3.08-3.17(\mathrm{~m}, 4 \mathrm{H})$, $4.94(\mathrm{br} \mathrm{s}, 2 \mathrm{H}), 5.77\left(\mathrm{q},{ }^{3} J_{\mathrm{HH}}=6.5 \mathrm{~Hz}, 2 \mathrm{H}\right), 7.10\left(\mathrm{dd},{ }^{3} J_{\mathrm{HH}}=5.1 \mathrm{~Hz},{ }^{4} J_{\mathrm{HH}}=1.7 \mathrm{~Hz}, 2 \mathrm{H}\right), 7.26(\mathrm{~d}$, $\left.{ }^{4} J_{\mathrm{HH}}=1.7 \mathrm{~Hz}, 2 \mathrm{H}\right), 8.37\left(\mathrm{~d},{ }^{3} J_{\mathrm{HH}}=5.1 \mathrm{~Hz}, 2 \mathrm{H}\right) ;{ }^{13} \mathrm{C}-\mathrm{NMR}\left(\mathrm{CDCl}_{3}, 75.5 \mathrm{MHz}\right): \delta 20.8\left(2 \mathrm{CH}_{3}\right)$, 
$26.5\left(\mathrm{CH}_{2}\right), 28.9\left(2 \mathrm{CH}_{2}\right), 29.2\left(2 \mathrm{CH}_{2}\right), 29.7\left(2 \mathrm{CH}_{2}\right), 40.9\left(2 \mathrm{CH}_{2}\right), 72.4(2 \mathrm{CH}), 120.6(2 \mathrm{CH})$, $122.7(2 \mathrm{CH}), 144.7$ (2C), $149.9(2 \mathrm{CH}), 155.4$ (2C), 162.8 (2C); $\mathrm{MS}\left(\mathrm{ESI}^{+}, \mathrm{m} / \mathrm{z}\right): 527$ $\left[\left(\mathrm{M}^{37} \mathrm{Cl}^{35} \mathrm{Cl}+\mathrm{H}\right)^{+}, 66 \%\right], 525\left[\left(\mathrm{M}^{35} \mathrm{Cl}^{35} \mathrm{Cl}+\mathrm{H}\right)^{+}, 100 \%\right]$; Anal. Calcd. (\%) for $\mathrm{C}_{25} \mathrm{H}_{34} \mathrm{~N}_{4} \mathrm{O}_{4} \mathrm{Cl}_{2}$ : C, 57.23; H, 6.54; N, 10.69. Found: C, 57.1; H, 6.5; N, 10.7.

\section{General procedure for the synthesis of dimers $(S, S)-4 a-c$}

In a sealed tube the corresponding dimer $(S, S)$-10a-c $(0.40 \mathrm{mmol})$ was dissolved in a $40 \%$ aqueous solution of dimethylamine $(1.00 \mathrm{~mL}, 8.00 \mathrm{mmol})$. The solution was stirred at $100{ }^{\circ} \mathrm{C}$ during $22 \mathrm{~h}$ until complete consumption of the starting material. The solvent was evaporated and the residue purified by flash chromatography affording the corresponding dimers $(S, S)-\mathbf{4 a - c}(91$ $92 \%)$.

$(1 S, 1 ' S)$-Bis[1-(4- $N, N$-dimethylaminopyridin-2-yl)ethyl]butane-1,4-diylbiscarbamate $(S, S)$ (4a). $92 \%$ isolated yield (white solid). $R_{f}(100 \% \mathrm{MeOH}): 0.40 ; \mathrm{Mp}: 138-140{ }^{\circ} \mathrm{C} ;[\alpha]_{\mathrm{D}}{ }^{20}=-34.3$ (c=2.0, $\mathrm{CHCl}_{3}$ ); IR (KBr): $v_{\max } 3348,1700,1654,1609,1560,1508,1261,1070 \mathrm{~cm}^{-1}$; ${ }^{1} \mathrm{H}-\mathrm{NMR}$ $\left(\mathrm{CDCl}_{3}, 300.13 \mathrm{MHz}\right): \delta 1.46-1.54(\mathrm{~m}, 4 \mathrm{H}), 1.56\left(\mathrm{~d},{ }^{3} J_{\mathrm{HH}}=6.6 \mathrm{~Hz}, 6 \mathrm{H}\right), 2.99(\mathrm{~s}, 12 \mathrm{H}), 3.13-3.22$ $(\mathrm{m}, 4 \mathrm{H}), 4.94(\mathrm{br} \mathrm{s}, 2 \mathrm{H}), 5.70\left(\mathrm{q},{ }^{3} J_{\mathrm{HH}}=5.9 \mathrm{~Hz}, 2 \mathrm{H}\right), 6.39\left(\mathrm{dd},{ }^{3} J_{\mathrm{HH}}=5.9 \mathrm{~Hz},{ }^{4} J_{\mathrm{HH}}=2.6 \mathrm{~Hz}, 2 \mathrm{H}\right)$, $6.51\left(\mathrm{~d},{ }^{4} J_{\mathrm{HH}}=2.6 \mathrm{~Hz}, 2 \mathrm{H}\right), 8.20\left(\mathrm{~d},{ }^{3} J_{\mathrm{HH}}=5.9 \mathrm{~Hz}, 2 \mathrm{H}\right) ;{ }^{13} \mathrm{C}-\mathrm{NMR}\left(\mathrm{CDCl}_{3}, 75.5 \mathrm{MHz}\right): \delta 20.9$ $\left(2 \mathrm{CH}_{3}\right), 27.2\left(2 \mathrm{CH}_{2}\right), 39.1\left(4 \mathrm{CH}_{3}\right), 41.0\left(2 \mathrm{CH}_{2}\right), 73.7(2 \mathrm{CH}), 103.2(2 \mathrm{CH}), 105.5(2 \mathrm{CH}), 149.3$ (2CH), 154.8 (2C), 155.9 (2C), 160.5 (2C); MS (ESI $\left.{ }^{+}, \mathrm{m} / \mathrm{z}\right): 473$ [(M+H) $\left.{ }^{+}, 100 \%\right]$; Anal. Calcd. (\%) for $\mathrm{C}_{24} \mathrm{H}_{36} \mathrm{~N}_{6} \mathrm{O}_{4}: \mathrm{C}, 61.00 ; \mathrm{H}, 7.68 ; \mathrm{N}, 17.78$. Found: C, 61.1; H, 7.7; N, 17.8.

$(1 S, 1 ' S)$-Bis[1-(4- $N, N$-dimethylaminopyridin-2-yl)ethyl]hexane-1,6-diylbiscarbamate $(S, S)$ (4b). 93\% isolated yield (colorless oil). $R_{f}(70 \% \mathrm{MeOH} / \mathrm{EtOAc}): 0.20 ;[\alpha]_{\mathrm{D}}{ }^{20}=-30.0(\mathrm{c}=2.0$, $\left.\mathrm{CHCl}_{3}\right)$; IR $(\mathrm{NaCl}): v_{\max } 3334,1715,1646,1560,1443,1376,1254,1136 \mathrm{~cm}^{-1}$; ${ }^{1} \mathrm{H}-\mathrm{NMR}$ $\left(\mathrm{CDCl}_{3}, 400.13 \mathrm{MHz}\right): \delta 1.19-1.29(\mathrm{~m}, 8 \mathrm{H}), 1.39-1.49(\mathrm{~m}, 4 \mathrm{H}), 1.54\left(\mathrm{~d},{ }^{3} J_{\mathrm{HH}}=6.2 \mathrm{~Hz}, 6 \mathrm{H}\right), 2.99$ $(\mathrm{s}, 12 \mathrm{H}), 3.05-3.18(\mathrm{~m}, 4 \mathrm{H}), 5.18(\mathrm{br} \mathrm{s}, 2 \mathrm{H}), 5.71\left(\mathrm{q},{ }^{3} J_{\mathrm{HH}}=6.8 \mathrm{~Hz}, 2 \mathrm{H}\right), 7.17\left(\mathrm{dd},{ }^{3} J_{\mathrm{HH}}=6.0 \mathrm{~Hz}\right.$, $\left.{ }^{4} J_{\mathrm{HH}}=2.4 \mathrm{~Hz}, 2 \mathrm{H}\right), 6.45\left(\mathrm{~d},{ }^{4} J_{\mathrm{HH}}=2.4 \mathrm{~Hz}, 2 \mathrm{H}\right), 8.12\left(\mathrm{~d},{ }^{3} J_{\mathrm{HH}}=6.0 \mathrm{~Hz}, 2 \mathrm{H}\right) ;{ }^{13} \mathrm{C}-\mathrm{NMR}\left(\mathrm{CDCl}_{3}\right.$, $100.6 \mathrm{MHz}): \delta 20.7\left(2 \mathrm{CH}_{3}\right), 26.2\left(2 \mathrm{CH}_{2}\right), 29.9\left(2 \mathrm{CH}_{2}\right), 39.4\left(4 \mathrm{CH}_{3}\right), 40.8\left(2 \mathrm{CH}_{2}\right), 71.8(2 \mathrm{CH})$, $103.8(2 \mathrm{CH}), 105.3(2 \mathrm{CH}), 149.5(2 \mathrm{CH}), 155.0$ (2C) 155.8 (2C), 160.9 (2C); MS (ESI $\left.{ }^{+}, \mathrm{m} / \mathrm{z}\right)$ : $501\left[(\mathrm{M}+\mathrm{H})^{+}, 100 \%\right]$; Anal. Calcd. $(\%)$ for $\mathrm{C}_{26} \mathrm{H}_{40} \mathrm{~N}_{6} \mathrm{O}_{4}: \mathrm{C}, 62.38 ; \mathrm{H}, 8.05 ; \mathrm{N}, 16.79$. Found: C, $62.3 ; \mathrm{H}, 8.1 ; \mathrm{N}, 16.7$.

(1S,1'S)-Bis[1-(4- $N, N$-dimethylaminopyridin-2-yl)ethyl]nonane-1,9-diylbis-carbamate $(S, S)$ (4c). $91 \%$ isolated yield (colorless oil). $R_{f}(60 \% \mathrm{MeOH} / \mathrm{EtOAc}): 0.26 ;[\alpha]_{\mathrm{D}}{ }^{20}=-36.5(\mathrm{c}=2.0$, $\left.\mathrm{CHCl}_{3}\right)$; IR (NaCl): $v_{\max }$ 3396, 2933, 1715, 1646, 1560, 1444, 1404, 1255, $1137 \mathrm{~cm}^{-1}$; ${ }^{1} \mathrm{H}-\mathrm{NMR}$ $\left(\mathrm{CDCl}_{3}, 300.13 \mathrm{MHz}\right): \delta 1.15-1.22(\mathrm{~m}, 10 \mathrm{H}), 1.33-1.42(\mathrm{~m}, 4 \mathrm{H}), 1.49\left(\mathrm{~d},{ }^{3} J_{\mathrm{HH}}=6.1 \mathrm{~Hz}, 6 \mathrm{H}\right)$, $2.94(\mathrm{~s}, 12 \mathrm{H}), 3.01-3.16(\mathrm{~m}, 4 \mathrm{H}), 5.11(\mathrm{br} \mathrm{s}, 2 \mathrm{H}), 5.64\left(\mathrm{q},{ }^{3} J_{\mathrm{HH}}=6.6 \mathrm{~Hz}, 2 \mathrm{H}\right), 6.34\left(\mathrm{dd},{ }^{3} J_{\mathrm{HH}}=5.1\right.$ $\left.\mathrm{Hz},{ }^{4} J_{\mathrm{HH}}=1.7 \mathrm{~Hz}, 2 \mathrm{H}\right), 7.26\left(\mathrm{~d},{ }^{4} J_{\mathrm{HH}}=1.7 \mathrm{~Hz}, 2 \mathrm{H}\right), 8.37\left(\mathrm{~d},{ }^{3} J_{\mathrm{HH}}=5.1 \mathrm{~Hz}, 2 \mathrm{H}\right) ;{ }^{13} \mathrm{C}-\mathrm{NMR}$ $\left(\mathrm{CDCl}_{3}, 75.5 \mathrm{MHz}\right): \delta 21.0\left(2 \mathrm{CH}_{3}\right), 26.8\left(\mathrm{CH}_{2}\right), 29.1\left(2 \mathrm{CH}_{2}\right), 29.5\left(2 \mathrm{CH}_{2}\right), 30.0\left(2 \mathrm{CH}_{2}\right), 39.3$ $\left(4 \mathrm{CH}_{3}\right), 41.2\left(2 \mathrm{CH}_{2}\right), 72.5(2 \mathrm{CH}), 103.4(2 \mathrm{CH}), 105.6(2 \mathrm{CH}), 150.0(2 \mathrm{CH}), 155.8(2 \mathrm{C}), 156.1$ (2C), $161.3(2 \mathrm{C})$; MS (ESI $\left.{ }^{+}, \mathrm{m} / \mathrm{z}\right): 543\left[(\mathrm{M}+\mathrm{H})^{+}, 100 \%\right]$; Anal. Calcd. (\%) for $\mathrm{C}_{29} \mathrm{H}_{46} \mathrm{~N}_{6} \mathrm{O}_{4}: \mathrm{C}$, 64.16; H, 8.55; N, 15.49. Found: C, 64.2; H, 8.5; N, 15.4. 


\section{Acknowledgements}

This work has been supported by the Spanish Ministerio de Ciencia e Innovación (MICINN, Project MEC-CTQ 2007-61126). V.G.-F. thanks MICINN for a personal grant (Ramón y Cajal Program).

\section{References}

1. (a) Litvinenko, L. M.; Kirichenko, A. I. Dokl. Akad. Nauk SSSR Ser. Chim. 1967, 176, 97. (b) Steglich, W.; Höfle, G. Angew. Chem. Int. Ed. 1969, 8, 981. (c) Steglich, W.; Vorbruggen, H.; Höfle, G. Angew. Chem. Int. Ed. 1978, 17, 569. (d) Berry, D. J.; DiGiovanna, C. V.; Metrick, S. S.; Murugan, R. Arkivoc 2001, (i), 201.

2. (a) Wurz, R. P.; Lee, E. C.; Ruble, J. C.; Fu, G. C. Adv. Synth. Catal. 2007, 349, 2345. (b) Vedejs, E.; Jure, M. Angew. Chem. Int. Ed. 2005, 44, 3974. (c) Dalko, P. I.; Moisan, L. Angew. Chem. Int. Ed. 2004, 43, 5138. (d) France, S.; Guerin, D. J.; Miller, S. J.; Lectka, T. Chem. Rev. 2003, 103, 2985. (e) Murugan, R.; Scriven, E. F. V. Aldrichimica Acta 2003, 36, 21.

3. (a) Fu, G. C. Acc. Chem. Res. 2004, 37, 542. (b) Fu, G. C. Acc. Chem. Res. 2000, 33, 412. (c) Ruble, J. C.; Latham, H. A.; Fu, G. C. J. Am. Chem. Soc. 1997, 119, 1492. (d) Ruble, J. C.; Fu, G. C. J. Org. Chem. 1996, 61, 7230.

4. (a) Duffey, T. A.; Shaw, S. A.; Vedejs, E. J. Am. Chem. Soc. 2009, 131, 14. (b) Busto, E.; Gotor-Fernández, V.; Gotor, V. Adv. Synth. Catal. 2006, 348, 2626. (c) Shaw, S. A.; Aleman, P.; Christy, J.; Kampf, J. W.; Va, P.; Vedejs, E. J. Am. Chem. Soc. 2006, 128, 925. (d) Poisson, T.; Penhoat, M.; Papamicaël, C.; Dupas, G.; Dalla, V.; Marsais, F.; Levacher, V. Synlett 2005, 2285. (e) Dálaigh, C. O.; Hynes, S. J.; Maher, D. J.; Connon, S. J. Org. Biomol. Chem. 2005, 3, 981. (f) Kawabata, T.; Stragies, R.; Fukaya, T.; Nagaoka, Y.; Schedel, H.; Fuji, K. Tetrahedron. Lett. 2003, 44, 1545. (g) Priem, G.; Pelotier, B.; McDonald, S. J. F.; Anson, M. S.; Campbell, I. B. J. Org. Chem. 2003, 68, 3844. (h) Shaw, S. A.; Aleman, P.; Vedejs, E.; J. Am. Chem. Soc. 2003, 125, 13368. (i) Priem, G.; Anson, M. A.; MacDonald, S. J.; Pelotier, B.; Campbell, I. B. Tetrahedron Lett. 2002, 43, 6001. (j) Jeong, K.-S.; Kim, S.H.; Park, H.-J.; Chang, K.-J.; Kim, S. K. Chem. Lett. 2002, 1114. (k) Kawabata, T.; Yamamoto, K.; Momose, Y.; Yoshida, H.; Nagaoka, Y.; Fuji, K. Chem. Commun. 2001, 2700. (1) Kawabata, T.; Nagato, M.; Takasu, K.; Fuji, K. J. Am. Chem. Soc. 1997, 119, 3169.

5. (a) Vedejs, E.; Chen, X. J. Am. Chem. Soc. 1997, 119, 2584. (b) Vedejs, E.; Chen, X. J. Am. Chem. Soc. 1996, 118, 1809.

6. See for example: (a) Detz, R. J.; Delville, M. M. E.; Hiemstra, H.; van Marsaaven, J. H. Angew. Chem. Int. Ed. 2008, 47, 3777. (b) Weis, M.; Waloch, C.; Seiche, W.; Breit, B. J. Am. Chem. Soc. 2006, 128, 4188. (c) Chi, K.W.; Addicott, C.; Kryschenko, Y. K.; Stang, P. J. J. Org. Chem. 2004, 69, 964. 
7. See for example: (a) González-Alvarez, A.; Alfonso, I.; Díaz, P.; García-España, E.; GotorFernández, V.; Gotor, V. J. Org. Chem. 2008, 73, 374. (b) Peña, C.; González-Sabín, J.; Alfonso, I.; Rebolledo F., Gotor, V. Tetrahedron 2008, 64, 7709. (d) Quijada, F. J.; Gonzalez-Sabín J.; Rebolledo, F.: Gotor, V. Tetrahedron: Asymmetry 2008, 19, 2589.

8. Gotor, V., Alfonso, I., García-Urdiales, E. Eds. Asymmetric Organic Synthesis with enzymes; Wiley-VCH: Weinheim, Germany, 2008.

9. Mataza, V. G.; Maduskuie, T. P.; Shapiro, H. S.; Hesp, B.; Snyder, D. W.; Aharony, D.; Krell, R. D.; Keith, R. A. J. Med. Chem. 1990, 33, 1781. (b) Firestone, R. A.; Pisano, J. M.; Falck, J. R.; McPhaul, M. M.; Krieger, M. J. Med. Chem. 1984, 27, 1037.

10. García-Alles, L. F.; V. Gotor, Tetrahedron 1995, 51, 307.

11. (a) Gotor-Fernández, V; Ferrero, M.; Fernández, S.; Gotor, V. J. Org. Chem. 1999, 64, 7504. (b) Gotor-Fernández, V.; Fernández, S.; Ferrero, M.; Gotor, V.; Bouillon, R.; Verstuyf, A. Bioorg. Med. Chem. 2004, 12, 5443.

12. (a) Busto, E.; Gotor-Fernández, V.; Gotor, V. Tetrahedron: Asymmetry 2006, 17, 1007. (b) Busto, E.; Gotor-Fernández, V.; Gotor, V. Tetrahedron: Asymmetry 2005, 16, 3427.

13. Opris, D. M.; Franke, P.; Schlüter, A. D. Eur. J. Org. Chem. 2005, 822. 